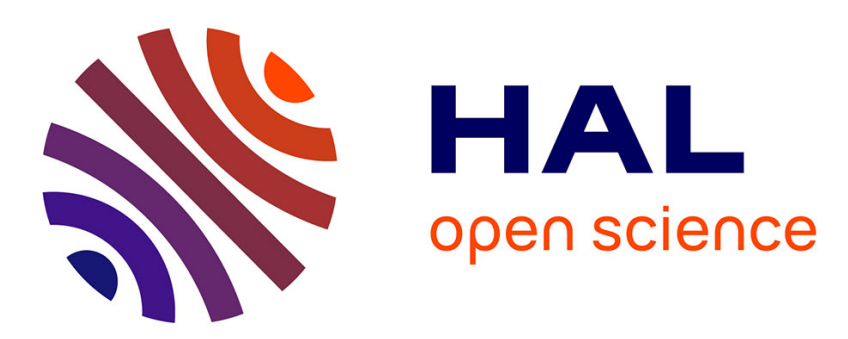

\title{
Passive Cooperative Targets for Subsurface Physical and Chemical Measurements: A Systems Perspective
} Jean Friedt

\section{To cite this version:}

Jean Friedt. Passive Cooperative Targets for Subsurface Physical and Chemical Measurements: A Systems Perspective. IEEE Geoscience and Remote Sensing Letters, 2017, 14 (6), pp.821 - 825. hal-02392623

\section{HAL Id: hal-02392623 \\ https://hal.science/hal-02392623}

Submitted on 4 Dec 2019

HAL is a multi-disciplinary open access archive for the deposit and dissemination of scientific research documents, whether they are published or not. The documents may come from teaching and research institutions in France or abroad, or from public or private research centers.
L'archive ouverte pluridisciplinaire HAL, est destinée au dépôt et à la diffusion de documents scientifiques de niveau recherche, publiés ou non, émanant des établissements d'enseignement et de recherche français ou étrangers, des laboratoires publics ou privés. 


\title{
Passive cooperative targets for subsurface physical and chemical measurements: a systems perspective
}

\author{
Jean-Michel Friedt
}

\begin{abstract}
We investigate the use of a commercially available Ground Penetrating RADAR (GPR) for probing the response of sub-surface sensors designed as passive cooperative targets. Such sensors must meet two design criteria: introduce a signature unique to the sensor which will hence be differentiated from clutter, and introduce in this signature a response characteristic allowing for recovering the physical quantity under investigation. Using piezoelectric substrates for converting the incoming electromagnetic pulse to an acoustic wave confined to the sensor surface (Surface Acoustic Wave transducer - SAW) allows for shrinking the sensor dimensions while providing sensing capability through the piezoelectric substrate acoustic wave velocity dependence with the physical quantity under investigation. Two broad ranges of sensing mechanisms are discussed: intrinsic piezoelectric substrate velocity dependence with a quantity restricted to the measurement of temperature or strain and hence torque or pressure - and extrinsic load dependence on the sensor, allowing for the measurement of variable capacitive or resistive loads. In all cases the delay introduced by the physical quantity variation induces a phase rotation of the returned signal of a few periods at most, to be measured with a resolution of a fraction of a period: the GPR receiver sampling time reference must exhibit a long term stability at least as good as the targeted phase measurement needed to recover the physical quantity. We show that the commercial GPR exhibits excessive time-base drift, yielding a loss in the sensing capability, while a quartz-oscillator based alternative implementation of the classical stroboscopic sampling receiver compensates for such a drawback.
\end{abstract}

Index Terms-surface acoustic wave, passive, wireless, sensor, ground penetrating radar, stability

\section{INTRODUCTION}

G ROUND Penetrating RADAR (GPR) cooperative targets [1] acting as passive sensors of sub-surface properties offer a complementary approach to mapping sub-surface dielectric and conductivity interfaces. In the cooperative target approach, the incoming electromagnetic pulse is delayed beyond clutter (Fig. 1), in our prefered implementation by converting the electromagnetic pulse to a $10^{5}$ times slower acoustic wave [2], and the returned signal delay is processed to recover the physical quantity. Clutter response, whose envelope including all returned signals from targets located at various distances from the emitter, is illustrated as the red envelope in Fig. 1: its power decays following the RADAR equation of Free Space Propagation Loss (FSPL) in which the emitted wave spreads energy on the surface of the propagating wavefront as $1 / d^{2}$, meets a point-like target acting itselft as the source of the spherical wave, resulting in the classical $1 / d^{4}$ power decay with distance $d$. Because the acoustic wave,

J.-M. Friedt is assistant professor at Franche-Comté University with his research activities hosted by the Time \& Frequency department of the FEMTO-ST Institute in Besançon, France. E-mail: jmfriedt@femto-st.fr.

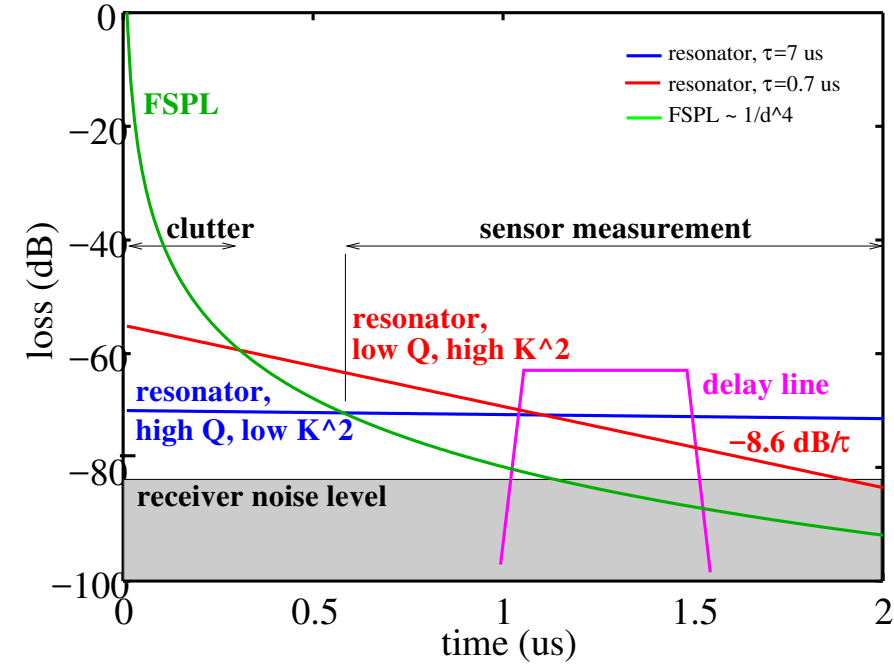

Fig. 1. Time dependence of the various backscattered signal, allowing for time-division multiple access to the radiofrequency channel: a short time window includes the envelope of echoes returned by all the buried interface reflections (green), while the sensor response is delayed beyond clutter in a longer time-window. In a resonator configuration, either a strongly coupled but lower $Q$ device (red) or a poorly coupled but high $Q$ (blue) allows for delaying the response above clutter at a time beyond $0.6 \mu$ s. Between 0.6 and $1 \mu \mathrm{s}$, the strongly coupled material provides a better link budget, above $1 \mu \mathrm{s}$ the weakly coupled material provides the best solution. The assumptions in drawing this chart is that the operating frequency is $f=100 \mathrm{MHz}$ so that the link budget at $300 \mathrm{~ns}(d=100 \mathrm{~m}$ in air $)$ is $20 \log _{10}(f d / c)=60 \mathrm{~dB}$, the clutter RADAR cross section is $1 \mathrm{~m}^{2}$, and the resonator coupling coefficient and quality factor are $K^{2} \simeq 0.03 \% \Rightarrow 20 \log _{10}\left(3 \cdot 10^{-4}\right)=-70 \mathrm{~dB}$ and $Q=2200$, or $K^{2} \simeq 1 \% \Rightarrow 20 \log _{10}\left(1 \cdot 10^{-2}\right)=-40 \mathrm{~dB}$ and $Q \simeq 220$ - the larger the coupling coefficient the lower the quality factor[6].

propagating at the air-piezoelectric substrate interface to meet boundary conditions of the acoustic field, exhibits a velocity dependence on such quantities as temperature [3], stress [4] or mass-loading [5], the returned signal delay will be dependent on those quantities, with the sensor design stage aiming at selecting a piezoelectric substrate orientation and packaging emphasizing one of these quantities.

The delay between the incoming radiofrequency pulse and returned signal is either generated by loading energy in a cavity which only slowly unloads once the excitation is completed - a property of resonators with a power release time constant of $\tau=Q /(\pi \cdot f)$ with $Q$ the quality factor and $f$ the resonance frequency [7], or by patterning mirrors on the surface of a piezoelectric transducer propagating the pulse converted from electromagnetic to acoustic [8]. The former approach is poorly suited to the broadband GPR which records hardly enough periods of the (exponentially decaying) returned signal to accurately identify $f[9]$, and the latter delay line method appears best suited when designing a GPR cooperative target. By using a differential approach, with two echoes returned by the sensor, the time of flight from the GPR unit to the buried 
sensor is eliminated, and an accurate estimate of the acoustic velocity is achieved by comparing the time of arrival of one echo with respect to the other. Such a comparison is classically performed by cross-correlation [10], in this example by crosscorrelating the two time-windows including each one of the two echoes returned by the cooperative target.

The core assumption in extracting a precise acoustic velocity estimate from the echo delays is that the sampling rate is constant. Indeed, typical acoustic transducer velocity dependence with temperature are at most in the $100 \mathrm{ppm} / \mathrm{K}$ : assuming a $100 \mathrm{MHz}$ transducer delaying the echo by 1 to $1.5 \mu \mathrm{s}$ - enough to get rid of clutter and yet prevent excessive acoustic losses while propagating on the substrate surface [11] - then the time difference of $1.5-1.0=0.5 \mu \mathrm{s}$ is associated with $0.5 \mu \mathrm{s} / 0.01 \mu \mathrm{s}=50$ complete phase rotations. Since the phase $\varphi$ and velocity $v$ variations are related by $\frac{d \varphi}{\varphi}=\frac{d v}{v}$, then a numerical application with the given values of $\frac{d v}{v} \simeq 100 \mathrm{ppm} / \mathrm{K}$ and $\varphi \simeq 50 \times 2 \cdot \pi$ yields $d \varphi \simeq 0.03 \mathrm{rad} / \mathrm{K}=1.8^{\circ} / \mathrm{K}$. This targeted accuracy of $1 / 200$ th of a period for $1 \mathrm{~K}$ resolution requires that the sampling rate varies by no more than this targeted resolution, otherwise echo analysis cannot differentiate between time of flight variations (observed as phase shift) or sampling rate variations. This topic is addressed here when using a Commercial, Off The Shelf (COTS) RADAR system for probing acoustic transducers acting as cooperative targets. The experiments reported throughout this paper have been acquired with a Malå ProEx unit running either the proprietary software provided by the manufacturer (Groundvision) or custom software specifically designed for sensing applications [12].

\section{ACOUSTIC TRANSDUCERS AS GPR COOPERATIVE TARGETS FOR PASSIVE SENSING}

Two approaches for using acoustic transducers as passive cooperative targets for remote sensing use either intrinsic material property dependence with the environment of the sensor - temperature or stress dependence of the electromechanical coefficients [13] combined with dilatation and deformation of the substrate subject to such effects - or connect an external load to the acoustic mirrors [14]. Such an external load impedance variation with a quantity - e.g. capacitance variation with moisture [15] when considering a capacitance architecture - induces a phase and amplitude variation on the reflected signal, readily detected on the echoes returned by the sensor. The challenging aspect of designing an external load is that matching impedance conditions must not prevent the incoming acoustic wave from being reflected: most demonstrations in the literature [15], [16] introduce such large amplitude variations of the returned signal that the interrogation range becomes strongly degraded.

In addition to the development of dedicated transducer for sensing purposes as discussed previously [17], we have considered commercially radiofrequency band-pass filters as suitable to this task [18]: indeed, very high frequency (VHF) filters are compact thanks to the conversion of the electromagnetic wave to an acoustic wave using a piezoelectric substrate most commonly quartz, lithium niobate or lithium tantalate
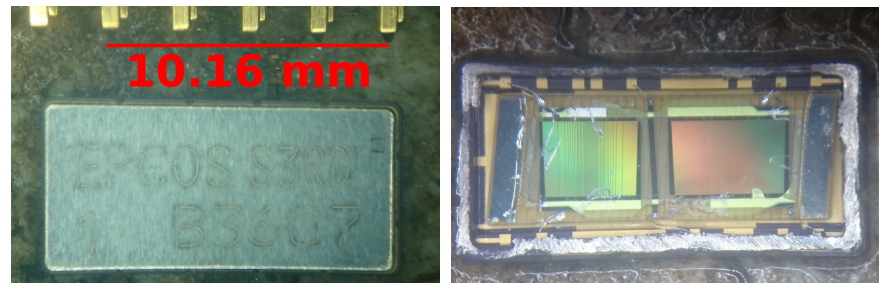

Fig. 2. Pictures of the closed (top) and opened (bottom) SAW filter: despite some scratches made on the electrodes while removing the polymer seal over the electrode area, the general structure of the filter with the input and output interdigitated transducer electrodes is visible.

- and hence exhibit suitable properties. Such VHF filters are traditionally characterized in transmission mode $\left(S_{21}\right)$ but are here considered as reflective transducers $\left(S_{11}\right)$ with either a fixed impedance on the second port inducing a strong reflected signal due to impedance mismatch, with a delay dependent on the acoustic velocity and hence the physical environment of the transducer, or a variable impedance inducing a phase rotation on the returned echo. The constraint on selecting the appropriate transducer are on the one hand in the frequency domain - the filter central frequency must match the GPR pulse characteristics but more significantly the inverse of the filter bandwidth must match the GPR pulse duration for efficient coupling - and in the time domain the group delay of the filter must be within acceptable measurement durations for a GPR, ideally in the 1 to $2 \mu$ s range, but being twice the tabulated group delay given in transmission mode in the manufacturer's datasheet, practically closer to the 2 to $4 \mu$ s range when the filter is used in reflection mode. As an example of such an application, the TDK/Epcos B3607 (Fig. 2) filter exhibits an appropriate response: its center frequency of $140 \mathrm{MHz}$ is compatible with a Malå $200 \mathrm{MHz}$ unshielded dipole antenna located on a concrete slab, and its $6 \mathrm{MHz}$ bandwidth induces a $150 \mathrm{~ns}$ returned echo duration, compatible with typical GPR measurement time windows. Fig. 3 demonstrates the measurement of a capacitive load, which was selected to demonstrate a load variation representative of classical Time Domain Reflectometry (TDR) moisture level measurement conditions. In this demonstration, the load circuit is tuned through an inductor selected to be resonant at $140 \mathrm{MHz}$ with the capacitance. Recovering the capacitance value is demonstrated through the filter reflection coefficient: on top of Fig. 3, the amplitude is hardly affected by the load variation with the returned echo delayed by $2.7 \mu \mathrm{s}$, while the phase (bottom graph) rotates by $120^{\circ}$ a 14 to $63 \mathrm{pF}$ load capacitance variation. In this example simulating a moisture detector in which soil permittivity is assumed to evolve from $\varepsilon_{r} \simeq 5$ when dry to $\varepsilon_{r} \simeq 22$ when wet or a 4.5 -fold relative permittivity rise, measuring the returned echo phase with sub$30^{\circ}$ accuracy is again mandatory.

Fine time delay analysis is best performed as a crosscorrelation between adjacent echoes. Since a cross-correlation of a periodically varying signal is itself varying with the same period, the technique is sensitive to $2 \pi$ phase rotation uncertainties. Furthermore, while cross-correlation in the time domain is an algorithm whose complexity rises as the square of the number of samples, the classical implementation is to reach the Fourier domain in which the cross-correlation becomes the dot product of the Fourier transforms, an algorithm requiring 

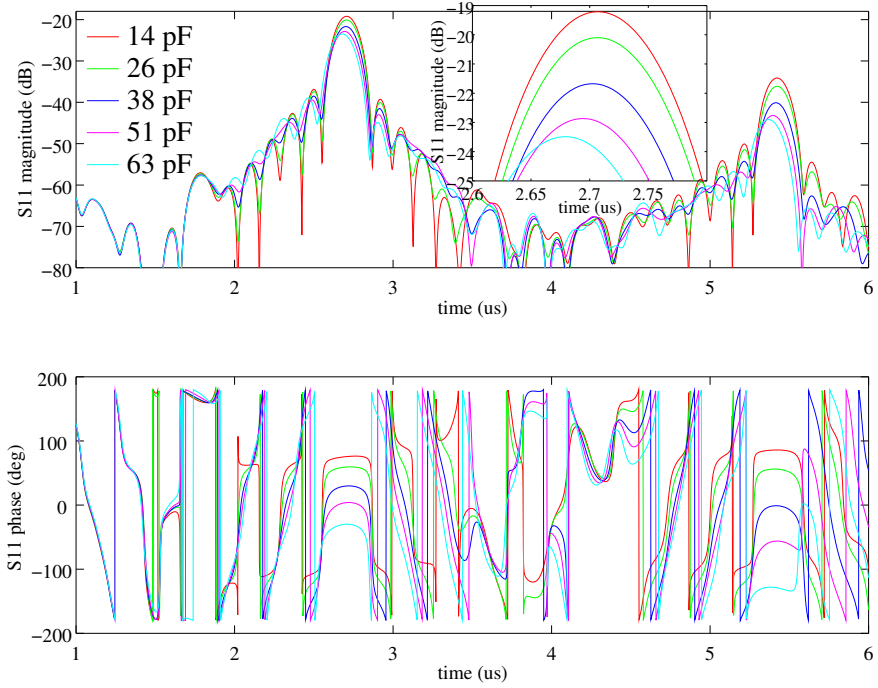

Fig. 3. Magnitude (top) and phase (bottom) of the time-domain response of the filter used in reflection mode and loaded with a variable capacitance in parallel to a fixed $10 \mathrm{pF}$ capacitance. The sum of the capacitances is given in the legend on the top left of the top graph, the color coding scheme being also valid for the bottom graph and the inset. Inset: zoom on the magnitude of the first echo, emphasizing the losses confined to the 19 to $24 \mathrm{~dB}$ range over the whole capacitance range.

only a $N \cdot \log (N)$ complexity thanks to the Fast Fourier Transform (FFT). Hence, analyzing the difference of the phase of the Fourier transform at its maximum power provides a fine estimate of the lag between adjacent echoes [19] and, considering the fixed geometrical path over which the acoustic wave propagates, a fine measurement of the acoustic velocity. Such a fine analysis will however emphasize the poor stability of GPR receiver time base with respect to current frequency references.

\section{COMmercial GPR MEASUREMENT OF ACOUSTIC TRANSDUCERS}

Experimental measurements have been performed as follows: a set of $250 \mathrm{MHz}$ shielded Malå antennas were elevated about $10 \mathrm{~cm}$ above concrete using two wooden blocks in order to locate an acoustic transducer connected to a dipole antenna with a polarization parallel to the GPR antennas (Fig. 4).

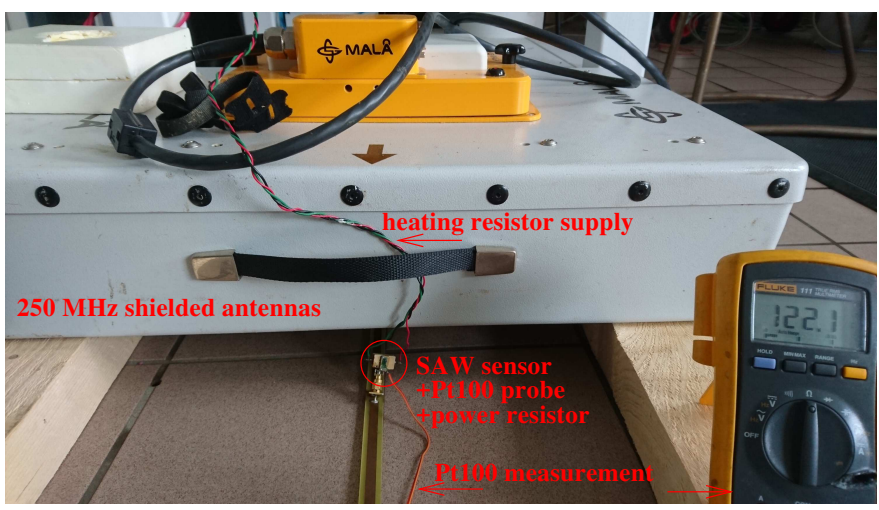

Fig. 4. Experimental setup including a sensor connected to a dipole antenna operating in the $250 \mathrm{MHz}$ range, and the Maå ProEx GPR unit fitted with shielded $250 \mathrm{MHz}$ antennas.

In order to optimize the transducer signal when illuminated by the GPR, the transducer is no longer a commercial filter
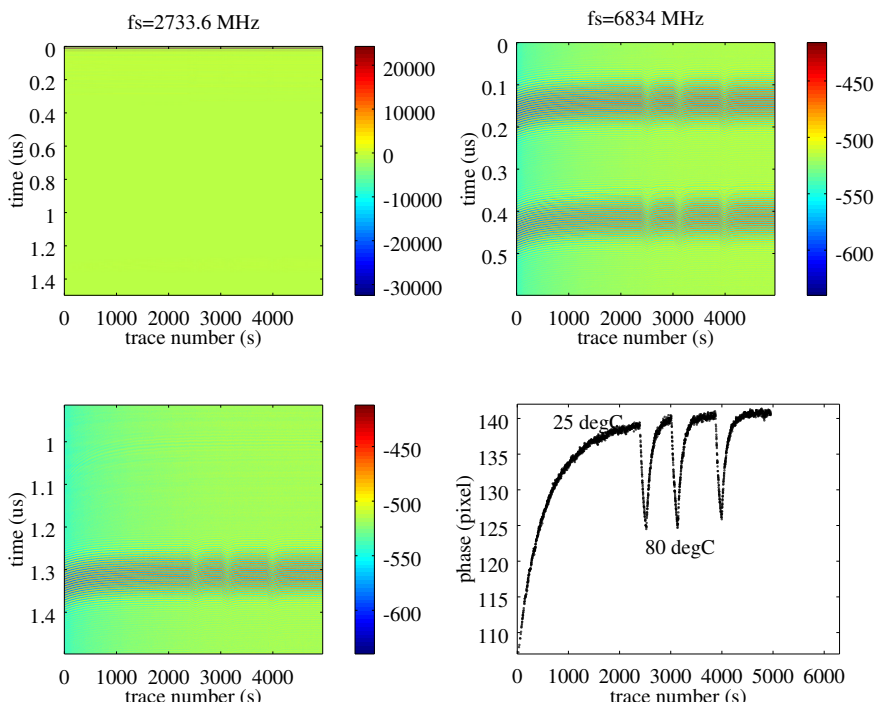

Fig. 5. Left: low sampling rate of the whole GPR trace (top left) and zoom on the echo returned by the sensor (bottom left), acquired using the proprietary Malå Groundvision software. Right: window centered on the two echoes returned by the sensor, recorded with the custom ProexGPRcontrol software (right), and phase difference extracted as the cross-correlation maximum position.
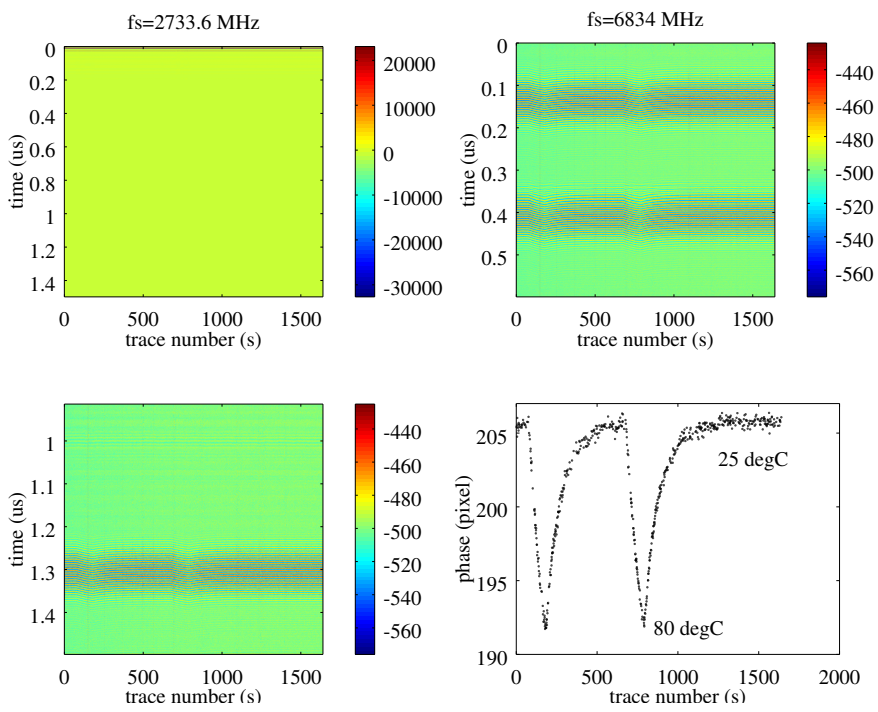

Fig. 6. Measurement of the same temperature sensor after the time-reference of the sampling stage has stabilized.

diverted from its original purpose but a custom delay line made of lithium niobate $\mathrm{YXl} / 128^{\circ}$, a crystalline orientation well known for propagating a Rayleigh wave exhibiting strong coupling and high temperature sensitivity $(70 \mathrm{ppm} / \mathrm{K})$, two properties suitable for a cooperative target for measuring sub-surface temperature. The design of this transducer was described in detail in [17] and will be used to gather the data shown in Figs. 5 to 8.

The GPR unit is switched on, and measurements start right after the software configuration setup is complete, requiring at most a few minutes. While we are well aware of the electronic unit warming up during the initial minutes, Figs. 5 exhibit slow drift for well above an hour, a significant hindrance when measuring on the field with a unit running on batteries. Figs. 5 and 6 (bottom right) exhibit the difference of the phases of the Fourier transform at its maximum amplitude, a fine 

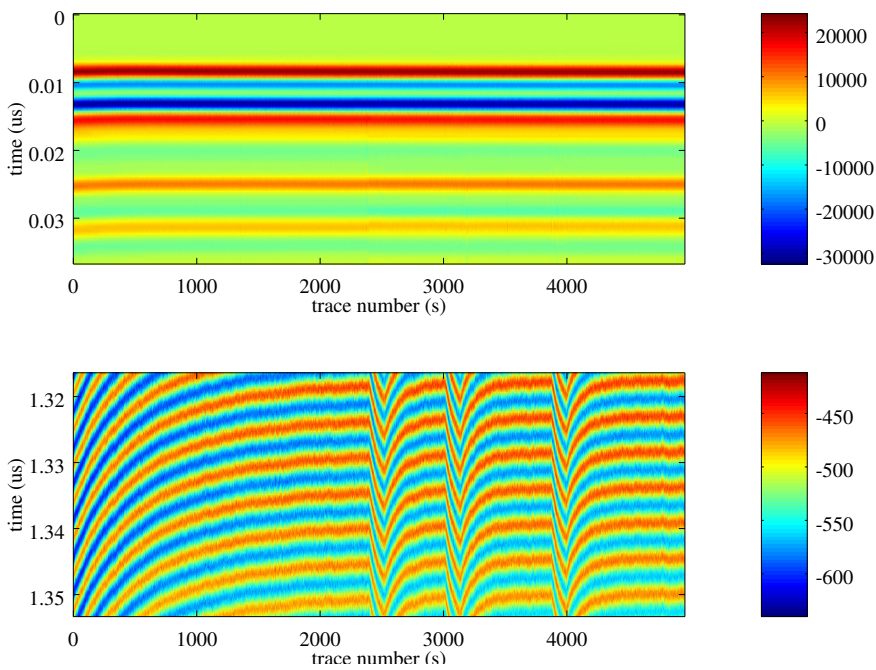

Fig. 7. Two time windows of the acquisition emphasizing the lack of drift on the position of the emitted pulse (top), and the sampling rate associated drift in the returned signal position after $1.33 \mu \mathrm{s}$.

measurement of the lag between the two echoes returned by the sensor. We have verified that a second-order polynomial fit on the cross-correlation magnitude of the two real signals, or the phase of the Fourier transform at the bin at which maximum power is measured (in the frequency domain) yield consistent results. In the time domain, a cross correlation on samples acquired with sampling step $t_{s}$ yields a time resolution on the echo position delay of $t_{s}$, which is then oversampled by using a parabolic fit on the cross-correlation magnitude. Such an oversampling strategy allows for reducing the delay uncertainty to a fraction of $t_{s}$ dependent on the signal to noise ratio. In the charts shown in Figs. 5 and 6 (bottom right), the sampling rate being $6834 \mathrm{MHz}$, the phase lag given as an ordinate scale is converted to a time lag by selecting $t_{s}=1 / 6834 \mu \mathrm{s}=146 \mathrm{ps}$ and 15 sampling interval lags last $2.2 \mathrm{~ns}$ or about half a period of a $250 \mathrm{MHz}$ signal. Hence, the drift during warm up observed in Fig. 6 of 30 sampling time intervals is equal to $4.4 \mathrm{~ns}$ or one full period of the $250 \mathrm{MHz}$ signal. While these measurements are performed, a Pt100 probe glued to the sensor is used to check that the room temperature does not vary by more than $1{ }^{\circ} \mathrm{C}$ during this time interval. One full phase rotation would have needed, on our sensor with $70 \mathrm{ppm} / \mathrm{K}$ sensitivity, a temperature variation of $d \varphi / \varphi=70 \cdot 10^{-6} \times 1 / d T \Rightarrow d T=(1 / 325) \cdot\left(1 / 70 \cdot 10^{-6}\right)=$ $44^{\circ} \mathrm{C}$ since the phase rotation of a $1.3 \mu$ s echo delay on a $250 \mathrm{MHz}$ delay line is $1.3 \times 250=325$ periods.

Reaching a stable asymptotic condition, three current pulses power a $5 \Omega$ power resistor to heat the sensor to $80{ }^{\circ} \mathrm{C}$ as observed on the Pt100 probe. Having reached this temperature, the sensor is let to cool back to room temperature before the next heating step starts (Fig. 5). The experiment is repeated (Fig. 6) after the GPR unit has stabilized after more than 2 hours of operation: here no significant drift of the baseline is observed and the two heating steps to $80{ }^{\circ} \mathrm{C}$ are readily identified.

Rather than focus on the processed data, the raw RADARgram already exhibits such a drift: Fig. 7 focuses on the emitted pulse (top) and the returned echo (bottom). Because the acoustic delay line transducer is made of an optimum number of electrode pairs of 20 (equal to the inverse of the electromechanical coupling coefficient [11]), a single incoming echo induces 20 periods in the returned echo, as seen on Fig. 7 (bottom). The initial drift between traces 0 and 1000 , followed by the slow stabilization prior to the three heating steps (traces 2500, 3000 and 4000), is well visible while the emitted pulse position remains stable throughout the experiment.

\section{DISCUSSION}

The excessive time-base drift requires either to increase the sensor sensitivity, or to improve the time base stability. The former approach requires for example to increase the time delay between the reflected echoes, with the drawback of additional acoustic losses (which remains hardly an issue at sub-GHz frequencies) and increased sensor dimensions. Most dramatic though is the physical limitation in some measurement strategies which prevent high sensitivity: in the case of gravimetric measurements for detecting chemical compounds in sub-surface environments, typical gravimetric sensitivities $S$ relating the relative velocity shift to the mass $\Delta m$ adsorbed per unit area $A$ are $S=\frac{d v}{v} \cdot \frac{A}{\Delta m} \simeq 200 \mathrm{~cm}^{2} / \mathrm{g}$ at most, so that a detection limit given by $\frac{\Delta m}{A}=\frac{\Delta \varphi}{\varphi} \cdot \frac{1}{S}=25 \mu \mathrm{g} / \mathrm{cm}^{2}$, well above the targeted detection limit of a few tens of nanograms par square centimeters in most applications.

Despite being well suited to sensor probing thanks to the ability to define the time-offset of various time-windows, either for sub-surface interface probing or sensor interrogation, stroboscopy must not degrade the sampling rate stability. Although the detailed implementation by Malå of their stroboscopic receiver is described in [20], we base our analysis on our own implementation attempt [21] in which the variable delay is coarsely set by an FPGA and finely tuned by a programmable delay line, in our case Maxim DS1023. Since this particular component stability with environment, and most significantly temperature, is not characterized by the manufacturer, we consider the information provided by Data Delay Devices Inc. in their 3D3418 series programmable delay lines in which a $\pm 3 \%$ stability is given over the $0--70^{\circ}$ temperature range. Such a percent range stability, or $10000 \mathrm{ppm}$ if $1 \%$ is considered, is well below expected stabilities for high quality acquisition systems, with quartz oscillators commonly exhibiting stability in the few tens of ppm, or a thousand fold stability improvement. Hence, the alternative is to replace the poor sampling rate stability generated by the programmable delay lines with a quartz-stabilized sampling rate. Such a solution was proposed in [22], and indeed yields acceptable sensor resolution with, for example, a gravimetric detection limit lowered to a few tens of nanograms per square centimeter, consistent with classical biosensor detection limits.

In addition to these considerations focusing on the receiver stage, another cause of concern is on the emitter: the avalanche transistor [23] feeding the antenna with a single powerful pulse in the pulsed RADAR approach introduces a possible variability on the emitted spectrum (Fig. 8). Indeed, while the pulse duration is determined by the high-voltage capacitance accumulating charges on the collector of the avalanche transistor before the next pulse is triggered by polarizing the base, 


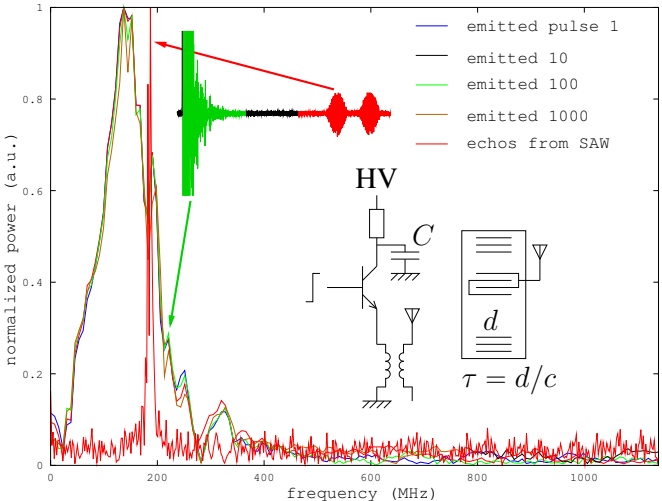

Fig. 8. Emitted spectra recorded over 35 minutes (green region of the time domain inset curve), and spectrum of the signal returned (red region of the time domain inset curve) by the sensor in a constant environment: no visible evolution of the emitted or returned signal is visible over this time span, confirming the cause of the phase drift as being due to the stroboscopic receiver timebase.

the antenna connected to the transistor emitted through a balun acts as a filter of the broadband pulse. The transfer function of the antenna is strongly dependent on the permittivity of its environment [24]: even shielded antennas are in close contact with the soil and permittivity variations will introduce variations in the emitted pulse spectrum. However because the acoustic wave propagation introduces a phase given by the acoustic path length to the wavelength ratio, varying the pulse spectrum will vary the phase rotation introduced by a given path length and assuming a constant acoustic velocity. Because wet to dry soil will induce drastic permittivity changes, the loaded dipole central frequency can vary by up to $50 \%$ and so will the emitted pulse spectrum. Such effects are readily observed when performing GPR measurements on wet snow, where permittivity strongly varies from rock to ice to snow to water soaked snow.

\section{CONCLUSION}

We have addressed the issue of stability of the receiver stage of stroboscopic sampler of a commercial Ground Penetrating RADAR (GPR) when probing the response of a cooperative target acting as a passive wireless sensor whose output information lies in the returned echo phase. While the echo delay is representative of the physical quantity under investigation, the phase variation associated with typical temperature, stress or mass loading variations is in the fraction of period range, while the sampling rate drift after the receiver is switched on is in the multiple period range. Since the sensor sensitivity is limited by the physics of acoustic wave propagation, the sensor response being delayed beyond clutter in a compact package by converting the electromagnetic to an acoustic wave through the polarization of a single crystal piezoelectric substrate, the solution lies in providing a stable receiver time-base. By using a stroboscopic approach clocked by a quartz stabilized timebase, sufficient stability is reached for probing passive wireless sensors by a GPR instrument.

\section{ACKNOWLEDGMENT}

The Malå ProEx GPR control unit acquisition was funded by a grant from the Franche-Comté district. Part of these investigations were motivated by the CITEPH SMASH project.

\section{REFERENCES}

[1] C. Allen, K. Shi, and R. Plumb, "The use of ground-penetrating radar with a cooperative target," IEEE Transactions on Geoscience and Remote Sensing, vol. 36, no. 5, pp. 1821-1825, 1998.

[2] A. Pohl, "A review of wireless saw sensors," IEEE Transactions on ultrasonics, ferroelectrics, and frequency control, vol. 47, no. 2, pp. 317-332, 2000.

[3] L. Reindl, G. Scholl, T. Ostertag, H. Scherr, U. Wolff, and F. Schmidt, "Theory and application of passive saw radio transponders as sensors," IEEE transactions on ultrasonics, ferroelectrics, and frequency control, vol. 45, no. 5, pp. 1281-1292, 1998.

[4] R. Stoney, D. Geraghty, and G. E. O'Donnell, “Characterization of differentially measured strain using passive wireless surface acoustic wave (saw) strain sensors," IEEE Sensors Journal, vol. 14, no. 3, pp. 722-728, 2014.

[5] H.-K. Oh, W. Wang, K. Lee, C. Min, and S. Yang, "The development of a wireless love wave biosensor on $41^{\circ} \mathrm{YX} \mathrm{LiNbO}_{3}$," Smart Mater. Struct., vol. 18, p. 025008, 2009.

[6] R. Wang, A. Bhave, and K. Bhattacharjee, "High kt2q, multi-frequency lithium niobate resonators," in 26th IEEE International Conference on Micro Electro Mechanical Systems (MEMS), Taipei, 2013.

[7] L. Reindl, G. Scholl, T. Ostertag, C. Ruppel, W.-E. Bulst, and F. Seifert, "Saw devices as wireless passive sensors," in Ultrasonics Symposium, 1996. Proceedings., 1996 IEEE, vol. 1. IEEE, 1996, pp. 363-367.

[8] W.-E. Bulst, G. Fischerauer, and L. Reindl, "State of the art in wireless sensing with surface acoustic waves," IEEE Transactions on Industrial Electronics, vol. 48, no. 2, pp. 265-271, 2001.

[9] J.-M. Friedt, A. Saintenoy, S. Chrétien, T. Baron, E. Lebrasseur, T. Laroche, S. Ballandras, and M. Griselin, "High-overtone bulk acoustic resonator as passive ground penetrating RADAR cooperative targets," Journal of Applied Physics, vol. 113, no. 13, p. 134904, 2013.

[10] P. E. Howland, D. Maksimiuk, and G. Reitsma, "Fm radio based bistatic radar," IEE Proceedings-Radar, Sonar and Navigation, vol. 152, no. 3 , pp. 107-115, 2005.

[11] D. Morgan, Surface acoustic wave filters: With applications to electronic communications and signal processing. Academic Press, 2010.

[12] [Online]. Available: https://sourceforge.net/projects/proexgprcontrol/

[13] R. Bechmann, A. Ballato, and T. Lukaszek, "Higher-order temperature coefficients of the elastic stiffinesses and compliances of alpha-quartz," Proceedings of the IRE, vol. 50, no. 8, pp. 1812-1822, 1962.

[14] T. Laroche, G. Martin, W. Daniau, S. Ballandras, J.-M. Friedt, and J.-F. Leguen, "A coupled-mode filter structure for wireless transceiver-sensors using reactive loads," in IEEE IFCS, 2012, pp. 1-6.

[15] L. Reindl, C. Ruppel, A. Kirmayr, N. Stockhausen, M. Hilhorst, and J. Balendonck, "Radio-requestable passive SAW water-content sensor," IEEE Transactions on Microwave Theory and Techniques, vol. 49, no. 4, pp. 803-808, 2001.

[16] J. Lee, J. Oh, J. Jun, and J. Jang, "Wireless hydrogen smart sensor based on Pt/graphene-immobilized radio-frequency identification tag," ACS nano, vol. 9, no. 8, pp. 7783-7790, 2015.

[17] J.-M. Friedt, T. Rétornaz, S. Alzuaga, T. Baron, G. Martin, T. Laroche, S. Ballandras, M. Griselin, and J.-P. Simonnet, "Surface acoustic wave devices as passive buried sensors," Journal of Applied Physics, vol. 109, no. 3, p. 034905, 2011.

[18] J.-M. Friedt, "Passive cooperative targets for subsurface physical and chemical measurements," in GPR2016, Hong Kong, 2016.

[19] A. V. Oppenheim and J. S. Lim, "The importance of phase in signals," Proceedings of the IEEE, vol. 69, no. 5, pp. 529-541, 1981.

[20] B. Johansson, "Ground penetrating radar array and timing circuit," 2002 , patent US 6496137.

[21] N. Chrétien, J.-M. Friedt, G. Martin, and S. Ballandras, "A stroboscopic approach to surface acoustic wave delay line interrogation," in Joint EFTF/IFCS. IEEE, 2013, pp. 771-774.

[22] F. Minary, D. Rabus, G. Martin, and J.-M. Friedt, "Note: A dualchip stroboscopic pulsed radar for probing passive sensors," Review of Scientific Instruments, vol. 87, no. 9, p. 096104, 2016.

[23] J. Williams, "High speed amplifier techniques," pp. 93-95, August 1991.

[24] C. A. Balanis, Antenna theory - analysis and design, 2nd Ed. John Wiley \& Sons, Inc, 1997.

Jean-Michel Friedt obtained his $\mathrm{PhD}$ in 2000 after working on biosensors and scanning probe microscopy. He became involved in the use of acoustic transducers as passive wireless sensors when he joined the company SENSeOR as a systems engineer in 2006, and with GPR as a tool for mapping a polar glacier thickness and subsurface features in Spitsbergen. Both topics appeared perfectly matched, becoming a core research activity since he joined the Franche-Comté University in Besançon, France, in 2014 as assistant professor. 